\title{
Binder use obscures traumatic pelvic injury in a 29-year-old man
}

\author{
K.T. Matthew Seah MRCSEd, James W. MacKay PhD
}

Cite as: CMAJ 2021 February 8;193:E216. doi: 10.1503/cmaj.201114

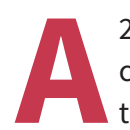

29-year-old male motorcyclist was brought to a trauma centre after colliding with a highway barrier. The prehospital team had applied a pelvic binder, given the patient's hemodynamic instability. The patient's heart rate before admission to hospital was 144 beats/min, respiratory rate was 28 breaths/ min and blood pressure was $100 / 65 \mathrm{~mm} \mathrm{Hg}$. In the emergency department, the patient was alert and reported lower abdominal discomfort. On examination, his abdomen was soft and he had bilateral open lower-limb fractures with suspected vascular injury. A trauma computed tomography (CT) scan showed no intra-abdominal injury or pelvic fracture, but did show a pelvic hematoma with no signs of active bleeding (Appendix 1, available at www. cmaj.ca/lookup/doi/10.1503/cmaj.201114/tab-related-content).

The patient was hemodynamically stable after initial resuscitation and the pelvic binder was removed. A routine pelvic radiograph taken after removal of the binder (in keeping with local guidelines) showed diastasis of the pubic symphysis and an injury to the left sacroiliac joint (Figure 1A). A retrospective review of the $\mathrm{CT}$ imaging while the binder was still in place showed some signs that may have indicated a more serious injury than initially suspected, such as the pelvic hematoma, a small avulsion fracture at the pubic symphysis (Figure 1B) and a subtle widening of the left sacroiliac joint on the initial CT imaging (Figure $1 \mathrm{C}$ ). ${ }^{1}$

Local pre-hospital guidelines state that a binder should be applied if there is suspicion of a pelvic injury after blunt high-energy trauma with hemodynamic instability (or systolic blood pressure $<110 \mathrm{~mm} \mathrm{Hg}$ ). ${ }^{2}$ Serious pelvic injuries may be missed in the presence of a well-applied pelvic binder, ${ }^{3}$ which is particularly true for purely ligamentous anteroposterior compression injuries. Trauma centres should also have a clear protocol for binder removal. ${ }^{4}$

This case reminds practitioners that a well-applied pelvic binder can sometimes mask ligamentous injuries in open-book pelvic fractures, and a plain radiograph of the pelvis should always be obtained after removing the binder in trauma patients.

\section{References}

1. Dreizin D, Nascone J, Davis DL, et al. Can MDCT unmask instability in binderstabilized pelvic ring disruptions? AJR Am J Roentgenol 2016;207:1244-51.

2. Scott I, Porter K, Laird C, et al. The prehospital management of pelvic fractures: initial consensus statement. Emerg Med J 2013;30:1070-2.

3. Clements $\mathrm{J}$, Jeavons $\mathrm{R}$, White $\mathrm{C}$, et al. The concealment of significant pelvic injuries on computed tomography evaluation by pelvic compression devices. $J$ Emerg Med 2015;49:675-8.

4. British Orthopaedic Association audit standards for trauma: the management of patients with pelvic fractures. London (UK): British Orthopaedic Association (BOA); 2018.

Competing interests: James MacKay reports receiving research support and consultancy fees from GlaxoSmithKline and GE Healthcare. No other competing interests were declared.

This article has been peer reviewed.

The authors have obtained patient consent.

Affiliations: Orthopaedic Trauma Unit (Seah), University of Cambridge, Addenbrooke's Hospital, Cambridge, UK; Department of Radiology (MacKay), Norwich Medical School, University of East Anglia, Norwich, UK

Content licence: This is an Open Access article distributed in accordance with the terms of the Creative Commons Attribution (CC BY-NCND 4.0) licence, which permits use, distribution and reproduction in any medium, provided that the original publication is properly cited, the use is noncommercial (i.e., research or educational use), and no modifications or adaptations are made. See: https://creativecommons.org/ licenses/by-nc-nd/4.0/

Correspondence to: K. Seah, matt.seah@doctors.org.uk 